\title{
CINÉTICA DE ANTICUERPOS CONTRA EL VIRUS DEL SÍNDROME REPRODUCTIVO Y RESPIRATORIO PORCINO DURANTE LAS ETAPAS DE RECRÍA, ENGORDE Y ACABADO EN UNA GRANJA DE LIMA
}

\author{
Kinetics of Antibodies against PRRSV in Weaning, Fattening and Finishing \\ Pigs in a Farm in Lima, Peru
}

\author{
Juan Calcina I. ${ }^{1}$, Hermelinda Rivera G, ${ }^{1,3}$, Mercy Ramírez V. ${ }^{1}$, Juan More B. ${ }^{1}$, \\ Gianfranco Arroyo H. ${ }^{1}$, Francisco Acosta C. ${ }^{2}$, Alberto Manchego S. ${ }^{1}$
}

\section{Resumen}

\begin{abstract}
El objetivo del presente estudio fue determinar la cinética de anticuerpos contra el virus del Síndrome Reproductivo y Respiratorio Porcino (VPRRS) en 30 animales de un lote de 200 de una granja porcina tecnificada de Lima. Se recolectaron muestras de sangre en tres periodos consecutivos a los 32, 61 y 136 días de edad para la determinación de anticuerpos contra el VPRRS mediante la prueba de ELISA indirecta. Adicionalmente, el lote de los 200 animales, incluidos los 30 animales experimentales, fue observado diariamente durante el tiempo que duró el estudio en busca de problemas respiratorios. El $26.7 \%$ (8/30) de las muestras de los lechones a los 32 días de edad tuvieron anticuerpos contra el VPRRS, así como una sola muestra a los 61 días de edad, mientras que el $96.7 \%$ (29/30) a los 136 días de edad presentaron anticuerpos contra el VPRRS con valores M/P entre 0.4 a 2.0. Durante el periodo de observación in situ de los animales del lote no se observaron signos respiratorios anómalos. Se encontró asociación significativa $(\mathrm{p}<0.05)$ entre la presencia de anticuerpos y la edad de los animales.
\end{abstract}

Palabras clave: porcino, anticuerpos, virus PRRS, ELISA

\section{Abstract}

The objective of the present study was to evaluate the kinetic of antibodies against the Porcine Reproductive and Respiratory Syndrome Virus (PRRSV) in 30 of a group of 200 pigs in a commercial farm in Lima, Peru. Blood samples were collected at 32, 61 and 135 days of age for detecting antibodies against PRRSV by indirect ELISA. In addition, the

\footnotetext{
${ }^{1}$ Laboratorio de Microbiología y Parasitología Veterinaria, Facultad de Medicina Veterinaria, Universidad Nacional Mayor de San Marcos, Lima

${ }^{2}$ San Fernando S.A., Lima

${ }^{3}$ E-mail: hriverag2005@yahoo.es
}

Recibido: 16 de abril de 2013

Aceptado para publicación: 21 de agosto de 2013 
occurrence of respiratory clinical signs was daily recorded in the total group of 200 animals including the 30 animals of the study. The $26.7 \%$ (8/30) of pigs had antibodies against PRRSV at 32 days of age and only one at 61 days of age, while $96.7 \%(29 / 30)$ had antibodies against PRRSV at 136 days of age with S/P values between 0.4 and 2.0. Non respiratory problems were recorded. The presence of antibodies and age of animals was statistically associated $(\mathrm{p}<0.05)$.

Key words: pigs, antibodies, PRRSV, ELISA

\section{INTRODUCCIÓN}

Se estima que la población porcina del Perú es de 4.7 millones (FAO, 2008), mayormente de tipo criollo distribuido a nivel nacional bajo el sistema de crianza de traspatio y familiar. Solo el $20 \%$ de esta población son animales de razas definidas bajo crianza intensiva, siendo los principales abastecedores de carne porcina al mercado nacional (Kalinowski, 2000; Arce et al., 2007).

La producción de carne porcina se ha incrementado en la última década, como lo demuestra el incremento del volumen de producción de 70 mil TM en 1998 a 114 mil TM en 2009 (MINAG, 2010). Este incremento de la producción se ve limitado por diversos factores, siendo uno de ellos las enfermedades infecciosas de origen bacteriano o viral como la peste porcina clásica, el síndrome reproductivo y respiratorio porcino (PRRS), circovirosis, etc., que afectan los sistemas reproductivo, respiratorio, nervioso e inmunitario del animal o que los predisponen a infecciones secundarias, incrementando las pérdidas económicas no solo por el alto costo del tratamiento de las infecciones secundarias, sino también por ser restrictivas para el comercio internacional (Van Reeth, 1996; Araínga et al., 2010).

La enfermedad del PRRS emergió en la población porcina de los EEUU a fines de la década del 80 y poco después en el continente europeo causando severas pérdidas económicas y caracterizada por desórdenes reproductivos en marranas, incrementada mortalidad perinatal y neumonías, y retardo en el crecimiento de los lechones (Neumann et al., 2005). El agente causal del PRRS fue identificado casi simultáneamente en Europa (Wensvoort et al., 1991) y en EEUU (Benfield et al., 1992). Desde entonces, numerosos estudios han demostrado que ambos virus pertenecen genómica y antigénicamente a genotipos distintos conocidos como genotipo 1 o europeo y genotipo 2 o americano (Meng et al., 1995; Shi et al., 2010). Actualmente, el PRRS es una enfermedad de gran importancia económica, sobre todo en países donde la porcicultura ha alcanzado gran desarrollo, superando incluso las pérdidas ocasionadas por enfermedades como peste porcina clásica y la enfermedad de Aujeszky (Neumann et al., 2005; Osorio, 2010).

En el Perú, el PRRS fue reportado serológicamente por Alegría et al. (1998). Desde entonces, estudios en campo evidencian la presencia del síndrome en granjas porcinas tecnificadas. El SENASA realizó un muestreo serológico en porcinos a nivel nacional en 2007 para identificar factores de riesgo asociados al PRRS y otras enfermedades y en 2010 se publicó un Decreto Supremo sobre el programa sanitario porcino, el cual da las pautas para el control de las enfermedades del porcino, entre ellas el PRRS (SENASA, 2011).

Recientemente se han aislado y genotipificado cepas del VPRRS de gorrinos de granjas porcinas de Lima y Arequipa con serología positiva al VPRRS, encontrándose que las cepas aisladas pertenecen al genotipo 1 (genotipo europeo). Un aspecto importante 
de ese estudio es que las muestras fueron obtenidas de animales sin signos clínicos de enfermedad, indicando el carácter subclínico de la infección (Ramírez et al., 2013).

Observaciones de campo y monitoreos serológicos indican que el VPRRS persiste en el grupo de animales en recría, engorde y acabado de algunas granjas porcinas, ocasionando problemas respiratorios y en concomitancia con otras infecciones como circovirus porcino $\mathrm{u}$ otros agentes bacterianos (Acosta F, 2011, Lima, comunicación personal). Sin embargo, en el país no existen informaciones de la dinámica de la infección dentro de estos grupos etarios que podría ser de importancia para tomar medidas de prevención del PRRS en la granja.

El presente estudio tuvo como objetivo determinar la cinética de anticuerpos contra el virus del PRRS en un lote de animales de una granja porcina tecnificada de Lima durante las fases de recría, engorde y acabado.

\section{Materiales y Métodos}

\section{Lugar de Estudio}

El estudio fue realizado en una granja porcina tecnificada ubicada en el valle de Lima que posee tres planteles destinados al área de maternidad y de recría, y un plantel final para el área de engorde. La granja es positiva a PRRS pero mayormente en el grupo de animales de engorde.

\section{Sistema de Manejo y Antecedentes Sa- nitarios}

La granja utiliza el sistema de manejo «todo dentro, todo fuera». El área de maternidad posee una infraestructura en piso de cemento y camas de paja proporcionando comodidad para la madre y los lechones. El área de recría consiste en galpones compuestos de paredes de madera y piso de arena con corrales para lotes de 200 animales, y el área de engorde posee una infraestructura de cemento.

El destete se realiza a los 24 días, donde los lechones pasan a los corrales de recría en lotes de 200. A los 77 días de edad son trasladados a los corrales de engorde y acabado, y entre los 130 y 140 días de edad son llevados al matadero.

$\mathrm{El}$ área de maternidad presentaba antecedentes de abortos, fetos momificados, nacidos débiles, lechones con problemas respiratorios y diarreas, causados aparentemente por el Circovirus tipo 2 y el virus del PRRS. En el grupo de animales de recría había antecedentes de diarreas y problemas respiratorios, y en el grupo de animales de engorde se presentaban problemas como úlceras gástricas, enteritis, neumonías y cuadros compatibles al síndrome de desmedro multisistémico posdestete. En un análisis previo en el lote de lechones de acabado y engorde se detectó una seroprevalencia del VPRRS mayor a $90 \%$.

\section{Animales y Muestras}

Se seleccionaron 30 lechones al azar sin importar sexo ni condición física (Dee y Joo, 1997) de un lote de 200 lechones, los cuales fueron identificados con aretes y permanecieron dentro del lote. La decisión de utilizar 30 animales se debió a problemas de logística. Adicionalmente, el lote de los 200 animales, incluidos los 30 animales experimentales, fue observado diariamente durante el tiempo que duró el estudio en busca de problemas respiratorios.

La obtención de muestras de sangre se hizo mediante punción de la vena cava anterior utilizando tubos al vacío. Las muestras fueron recolectadas a los 32, 61 y 136 días de edad, siendo la última muestra recolectada en el matadero durante el beneficio. El suero fue obtenido por centrifugación a $800 \mathrm{G}$ por 10 min y almacenado en viales a $-20^{\circ} \mathrm{C}$. 


\section{Detección de Anticuerpos contra VPRRS}

Los anticuerpos contra VPRRS fueron detectados mediante la prueba de ELISA indirecta de procedencia comercial (IDEXX, EEUU), siguiendo las indicaciones del fabricante. El resultado fue determinado calculando el coeficiente obtenido de la muestra sobre el coeficiente del control positivo (M/P). Una muestra de suero con coeficiente $\mathrm{M} / \mathrm{P}$ igual o mayor a 0.4 fue clasificada como positiva a anticuerpos contra el VPRRS.

\section{Análisis de Datos}

La frecuencia de animales positivos en cada etapa de muestreo fue expresada en porcentaje. La posible asociación estadística entre la variable edad de los animales con la variable presencia de anticuerpos se evaluó mediante el análisis de varianza.

\section{Resultados y Discusión}

En el Cuadro 1 se presentan los coeficientes M/P de las muestras analizadas a los 32, 60 y 136 días de edad. El $73.3 \%$ de las muestras recolectadas a los 32 días de edad no presentaron anticuerpos $(\mathrm{M} / \mathrm{P}<0.4)$. A los 61 días de edad, solo un animal fue seropositivo indicando que los anticuerpos detectados en los lechones a los 32 y 61 días de edad fueron anticuerpos pasivos. Por otro lado, el $96.7 \%$ de los gorrinos a los 136 días de edad presentaron altos niveles de anticuerpos en rangos de 0.4 a 2.0 .

Usualmente, los anticuerpos pasivos tienen una vida media de 12 a 14 días, pero pueden ser detectados hasta las 4 a 8 semanas de vida (Murtaugh et al., 2002; OIE, 2010). La no detección de anticuerpos en el $73.3 \%$ de lechones a los 32 días de edad indica que sus madres tuvieron bajo nivel o no tuvieron anticuerpos contra el VPRRS; es decir, posible ausencia de desafío viral activo en las marranas, ya que los anticuerpos pasivos guardan relación con los anticuerpos presentes en el suero de las madres. La no detec- ción de anticuerpos en la mayoría de los lechones a los 61 días de edad (Cuadro 1) significa que los animales estuvieron susceptibles a la infección por el VPRRS cuando fueron trasladados a los corrales de engorde a los 77 días de edad, siendo así que el $96.7 \%$ (29/30) se infectó con el VPRRS.

El análisis estadístico de los resultados indicó que hubo asociación entre las variables presencia de anticuerpos y edad de los animales ( $\mathrm{p}<0.05)$ (Cuadro 2). El VPRRS usualmente persiste en ambientes susceptibles como es el caso de animales de engorde y acabado.

La granja en estudio tiene el sistema de manejo «todo dentro, todo fuera». No obstante, pese al buen sistema de manejo y buena bioseguridad, el VPRRS llegó a ingresar a la granja y desde entonces la infección está siendo controlada en el área de marranas aunque no ha sido erradicada de la granja. En el lote de animales de recría, engorde y acabado son frecuentes las infecciones respiratorias y manifestaciones clínicas características del síndrome de Desmedro Posdestete causado por el Circovirus porcino tipo 2, por lo que los lechones fueron vacunados por primera vez contra este agente a los 32 días de edad, aprovechándose este manejo para la primera toma de muestras del presente estudio.

Durante el estudio, los problemas respiratorios y de mortalidad fueron escasos en los 200 animales del lote. Ninguno de los 30 animales muestreados murieron o presentaron signos respiratorios, a pesar de que la mayoría de ellos seroconvirtieron, al parecer, posterior a los 62 días de edad. La ausencia de signos respiratorios en el lote de animales en estudio pudo deberse a varios factores, entre ellos la adopción de estrictas medidas de bioseguridad y buenas prácticas sanitarias en los corrales de recría. Por otro lado, la endemicidad de la infección por PRRS usualmente asociada a cepas de baja virulencia podría ser debida a factores que contribuyen en la presentación subclínica del 
Cuadro 1. Distribución de los coeficientes M/P de las muestras positivas a anticuerpos contra el virus de Síndrome Reproductivo y Respiratorio Porcino en las tres etapas de muestreo $(n=30)$

\begin{tabular}{ccccccc}
\hline & \multicolumn{6}{c}{ Edad de los animales al muestreo } \\
\cline { 2 - 7 } Coeficiente M/P & \multicolumn{2}{c}{32 días } & \multicolumn{2}{c}{61 días } & \multicolumn{2}{c}{136 días } \\
\cline { 2 - 7 } & $\mathrm{n}$ & $\%$ & $\mathrm{n}$ & $\%$ & $\mathrm{n}$ & $\%$ \\
\hline$<0.4$ (seronegativos) & 22 & 73.3 & 29 & 96.7 & 1 & 3.3 \\
$>0.4$ Seropositivos & & & & & & \\
$0.4-0.8$ & 6 & 20.0 & 1 & 3.3 & 2 & 6.7 \\
$0.8-1.2$ & 0 & 0 & 0 & 0 & 6 & 20.0 \\
$1.2-1.6$ & 2 & 6.7 & 0 & 0 & 7 & 23.3 \\
$1.6-2.0$ & 0 & 0 & 0 & 0 & 14 & 46.7 \\
\hline Total & 8 & 26.7 & 1 & 3.3 & 29 & 96.7 \\
\hline
\end{tabular}

Cuadro 2. Elaboración del modelo lineal mixto para determinar el efecto de la edad de los animales sobre los niveles de densidad óptica de los anticuerpos contra el VPRRS

\begin{tabular}{lccccc}
\hline Variable & Coeficiente & $\begin{array}{c}\text { Error } \\
\text { estándar }\end{array}$ & $\mathrm{Z}$ & Prob $>|\mathrm{Z}|$ & IC (95\%) \\
\hline Edad & -0.7744 & 0.1014 & -7.34 & 0.0001 & $-0.943-0.5455$ \\
Edad 2 & 0.5770 & 0.0487 & 11.84 & 0.001 & $0.4812-0.6726$ \\
Intercepto & 0.2750 & 0.0435 & 6.31 & 0.001 & $0.1896-0.3604$ \\
\hline
\end{tabular}

PRRS en los corrales de engorde y acabado (Van Reeth, 1997; Mateu y Diaz, 2008; Shi et al., 2010).

Los anticuerpos detectados en las muestras a los 136 días de edad con un coeficiente $\mathrm{M} / \mathrm{P}$ entre 0.4 a 2.0 (Cuadro 1), indica un intenso desafío, dado que el $96.7 \%$ (29/30) de los gorrinos se infectaron y seroconvirtieron en un lapso de 2.5 meses. En una infección natural, los anticuerpos inducidos por el VPRRS aparecen entre 7 a 9 días de la infección, pero estos anticuerpos no tienen capacidad de neutralizar al virus (López y Osorio, 2004), por lo que el VPRRS persiste en la población en presencia de los anticuerpos. El $96.7 \%$ de seroprevalencia del VPRRS detectado en gorrinos durante el beneficio es similar a otros estudios cuyos autores reportan prevalencias de 70 a $100 \%$ en cerdos de engorde y acabado (Bøtner, 1997; Dee y Joo, 1997).

Si bien no se pudo establecer el momento exacto del inicio de la infección de los gorrinos con el VPRRS, se concluye que el virus es endémico en los corrales de engorde y acabado debido al continuo ingreso de animales susceptibles y que la infección es de tipo subclínica. 


\section{Agradecimiento}

El presente estudio fue financiado por el Consejo Superior de Investigaciones (CSI: código 110801151) de la Universidad Nacional Mayor de San Marcos, Lima. Perú.

\section{Literatura Citada}

1. Alegría ME, Rivera H, Manchego A. 1998. Evidencia del virus del síndrome reproductivo y respiratorio porcino de crianza tecnificada. Rev Inv Pec IVITA 9(1): 53-58.

2. Araínga M, Husanaga T, Hills $K$, Handel K, Rivera H, Pasick J. 2010. Phylogenetic analysis of classical swine fever virus isolates from Peru. Transbound Emerg Dis 57: 262-270.

3. Arce E, Alegre J, Escudero E, Prain G, Sáenz J. 2007. Crianza de cerdos en zonas urbanas: diagnóstico y propuesta municipal del sistema de manejo en el distrito de Lurigancho Chosica, Lima (Perú). En: Castro G (ed). Porcicultura urbana y periurbana en ciudades de América Latina y el Caribe. Perú: IPESRUAF. p 25-33.

4. Benfield DA, Nelson E, Collins JE, Harris L, Goyal SM, Robinson D, Christianson WT, et al. 1992. Characterization of swine infertility and respiratory syndrome (SIRS) virus (isolate ATCCVR-2332). J Vet Diagn Invest 4: 127-133.

5. Bøtner A. 1997. Diagnosis of PRRS. Vet Microbiol 55: 295-301.

6. Dee SA, Joo HS. 1997. Strategies to control PRRS: a summary of field and research experiencies. Vet Microbiol 55: 347- 353.

7. [FAO] Food and Agriculture Organization of the United Nations. 2008. Peste porcina clásica. [Internet], [13 septiembre 2011]. Disponible en: http://www.rlc.fao.org/es/prioridades/ transfron/ppc/peru.htm
8. Kalinowsky EJ. 2000. Situación de la porcicultura nacional: análisis y perspectivas. En: II Congreso Nacional de Porcicultura y Expo Porcino 2000. Lima. APPP. p 24.

9. López O, Osorio F. 2004. Role of neutralizing antibodies in PRRSV protective immunity. Vet Immunol Immunopathol 102: 155-163.

10. Mateu E, Diaz I. 2008. The challenge of PRS immunology. Vet J 177: 345-351.

11. Meng XJ, Paul PS, Halbur PG, Lum MA. 1995. Phylogenetic analysis of the putative $\mathrm{M}$ (Orf 6) and $\mathrm{N}$ (orf 7) genes of porcine reproductive and respiratory syndrome virus (PRRSV): implication for the existence of two genotypes of PRRSV in the USA and Europe. Arch Virol 140: 745-755.

12. [MINAG] Ministerio de Agricultura. 2010. Dinámica agropecuaria 1997 2009. Perú: MINAG-OEEE. p 22-25.

13. Murtaugh M, Xiao Z, Zuckermann F. 2002. Immunological responses of porcine reproductive and respiratory syndrome virus infection. Viral Immunol 15: 533-547.

14. Neumann E, Kliebenstain J, Mabry J, Bush E, Setziger A, Green A, Zimmermann J. 2005. Assessment of the economic impact of porcine reproductive and respiratory syndrome on swine production in the United States. JAVMA 227: 385-392.

15. Osorio F. 2010. PRRSV infections: a world-wide update. Acta Sci Vet 38 (Suppl 1): 269-275.

16. [OIE] World Organisation for Animal Health. 2010. OIE Terrestrial Manual. Porcine reproductive and respiratory syndrome. Ch 2.8.7. $4 \mathrm{p}$.

17. Ramírez M, Rivera H, Manchego A, More J, Chiok KL. 2013. Aislamiento y genotipificación del virus del síndrome respiratorio y reproductivo porcino (VPRRS) en granjas seropositivas de las provincias de Lima y Arequipa, Perú. Rev Inv Vet Perú 24: 222-232. 
18. [SENASA] Servicio Nacional de Sanidad Agraria. 2011. Lima [Internet], [20 abril 2011]. Disponible en: http:// www.senasa.gob.pe

19. Shi M, Tsan-Yuk Lam T, Hon CH, Kin Hei Hui R, Faaberg KS, WennblomT, Murtagh MP, et al. 2010. Molecular epidemiology of PRRSV: a phylogenetic perspective. Virus Res 154: 7-17.

20. Wensvoort G, Terpstra C, Pol JM, Ter Laak EA, Bloemraad M, De Kluyver EP; Kragten C, et al. 1991. Mystery swine disease in The Netherlnads: the isolation of Lelystad virus. Vet Quart 13: 121-130.

21. Van Reeth K. 1997. Pathogenesis and clinical aspects of a respiratory porcine reproductive and respiratory syndrome virus infection. Vet Microbiol 55: 223-230.

22. Van Reeth K, Narwuynck H, Pensaert M. 1996. Dual infections of feeder pigs with porcine reproductive and respiratory syndrome virus followed with porcine respiratory coronavirus on swine influenza virus. A clinical and virological study. Vet Microbiol 48: 325-335. 